\title{
ОПЫТ ПРИМЕНЕНИЯ МЕТОДОВ МАТЕМАТИЧЕСКОЙ СТАТИСТИКИ ДЛЯ ОЦЕНКИ СОСТОЯНИЯ СЕЛЬСКОХОЗЯЙСТВЕННЫХ РАСТЕНИЙ
}

\author{
В.М. БУРЕ 1,2 , А.Ф. ПЕТРУШИН 1 , Е.П. МИТРОФАНОВ1, \\ О.А. МИТРОФАНОВА 1,2, В. ДЕНИСОВ 3
}

Решение задач, связанных с оценкой состояния сельскохозяйственных растений в период вегетации, позволяет эффективно использовать удобрения, получать выгодную урожайность, улучшать качественные характеристики растений, а также экологическое состояние поля. Все более перспективным направлением в точном земледелии становится применение различных методов математической статистики. Нашей целью была оценка состояния сельскохозяйственных растений с использованием подхода, основанного на совместном применении методов кригинга и бинарной регрессии, а также определение обеспеченности посевов азотом по индексу NDVI (Normalized Difference Vegetation Index). Исследования проводили на участке опытного поля, расположенного на территории филиала Агрофизического института (д. Меньково, Ленинградская обл.) в 2015 году. С помощью аэрофотоснимков, сделанных с автоматизированного беспилотного летательного комплекса Геоскан-401 («Геоскан», Россия), был получен набор значений вегетационного индекса NDVI в произвольных точках участка. Также провели ряд наземных измерений. Предложенный подход к оценке состояния сельскохозяйственных растений заключался в совместном использовании двух методов математической статистики: ординарного кригинга и логистической регрессии. Осуществляли предварительный вариограммный анализ, выполняли построение модели вариограммы. После этого методом кригинга вычисляли ряд прогнозируемых значений исследуемого параметра. На следующем этапе устанавливали пороговое значение параметра для исследуемого участка, а также вводили фиктивную переменную, принимающую значение 1 , если величина параметра превышала порог, и 0 в противном случае. Затем строили логитмодель, в которой одним из факторов был ряд оценок интересующего параметра, полученных с использованием метода ординарного кригинга. Входные данные для построения логит-моделей были следующими: $N\left(x_{i}\right)-$ значение NDVI в местоположении $x_{i}, i=\overline{1,78}$; переменная $T=1$, если $N\left(x_{i}\right) \geq 0,46$, иначе $T=0$; переменные $X$ и $Y$ - координаты наблюдений, рассматриваются в качестве объясняющих переменных; $N_{\text {pred }}\left(x_{i}\right)$ - величины параметра, спрогнозированные с использованием метода кригинга в наблюдаемых точках. Все вычисления выполнялись с помощью языка программирования R. B результате проведенного эксперимента были построены три логитмодели с зависимой переменной $T$ : в первой модели объясняющие переменные $X$ и $Y$, во второй модели $-X, Y$ и $N_{p r e d}$, в третьей модели $-N_{\text {pred }}$. Тестирование показало, что при добавлении переменной $N_{\text {pred }}$ логит-модель работает лучше (в 2 раза меньше ошибочного определения исследуемого параметра). Полученные результаты дают основание полагать, что добавление в факторы бинарной регрессии набора спрогнозированных методом кригинга величин позволяет существенно повысить точность расчетов.

Ключевые слова: оценка состояния растений, Normalized Difference Vegetation Index, NDVI, кригинг, бинарная регрессия, язык R.

Оценка состояния сельскохозяйственных растений в период вегетации (обеспеченность питательными веществами, водный режим, сорняки, болезни и т.д.) необходима для эффективного применения удобрений, получения выгодной урожайности и высокого качества продукции (1-3). В последние годы для решения подобных задач все более перспективным становится статистический анализ, а также обработка данных дистанционного зондирования (4-6).

Один из новых подходов в агрофизике строится на методах, использующих бинарную регрессию. Например, в работе В.М. Буре (7) описано ее применение для прогноза урожайности. В исследованиях, проведенных в Норвегии и Нидерландах, предложены методики прогноза пространственного распределения типов почв на основе мультиномиальной логистической регрессии с использованием цифрового анализа местности $(8,9)$. К более изученным направлениям в точном земледелии относится геостатистика, которая позволяет картировать содержание в почве питательных веществ (азот, фосфор, калий и др.) $(10,11)$, а также электропро- 
водность, $\mathrm{pH}$, плотность, влажность (12-14) с целью оптимального управления хозяйством. При геостатистическом подходе почва рассматривается как набор переменных, непрерывных в пространстве, и их изменения описываются с точки зрения пространственной зависимости $(15,16)$. Сочетание методов геостатистики и бинарной регрессии предложено только в экономике (17), для задач точного земледелия подобные методологии не описаны.

В представленной работе мы впервые спрогнозировали пространственное распределение значений вегетационного индекса NDVI (Normalized Difference Vegetation Index) на опытном поле, применив регрессию на основе гауссовских процессов (кригинг) совместно с логистической регрессией как вариантом бинарной регрессии. При этом результаты тестирования показали, что предложенный подход достаточно точно оценивает интересующий параметр на исследуемом участке поля.

Нашей целью была характеристика состояния сельскохозяйственных растений с помощью сочетания методов кригинга и бинарной регрессии, а также определение обеспеченности посевов азотом (по индексу NDVI).

Методика. Исследования проводили на участке опытного поля на территории филиала Агрофизического института (д. Меньково, Ленинградская обл.) в 2015 году. С помощью аэрофотоснимков, сделанных с автоматизированного беспилотного летательного комплекса Геоскан-401 («Геоскан», Россия), был получен набор значений вегетационного индекса NDVI (Normalized Difference Vegetation Index) в произвольных точках участка (всего 78 значений).

Для анализа состояния сельскохозяйственных растений мы применяли совместно два метода математической статистики - ординарный кригинг и логистическую регрессию. При этом использовали логит-модель (logit-model) как подход, обеспечивающий простоту оценки параметров.

Пространственное распределение интересующего параметра прогнозировали с помощью ординарного кригинга для набора измерений $(18,19)$ :

$$
\hat{Z}\left(x_{0}\right)=\sum_{i=1}^{n} \lambda_{i} Z\left(x_{i}\right), \sum_{i=1}^{n} \lambda_{i}=1,
$$

где $n$ - число измерений, $Z\left(x_{i}\right)$ - значение наблюдаемого параметра в местоположении $x_{i}, \lambda_{i}-$ неизвестный вес для параметра, $\hat{Z}\left(x_{0}\right)-$ прогноз значения параметра в местоположении $x_{0}$.

Неизвестный вес находили с помощью вариограммного анализа с построением теоретической модели вариограммы $\gamma(h)$ на основании полученной экспериментальной кривой $\gamma(h)$.

Для выполнения логистической регрессии фиксировали значение $d$ (порог) для исследуемого участка поля и вводили фиктивную переменную:

$$
y(x)=\left\{\begin{array}{l}
1, Z(x) \geq d, \\
0, Z(x)<d .
\end{array}\right.
$$

При этом значения переменной $y\left(x_{i}\right)$ в наблюдаемых точках были известны, поскольку в этих точках известны величины интересующего параметра и его уровень относительно порогового значения. Кроме того, на предыдущем этапе мы получали набор спрогнозированных величин параметра с помощью кригинга. В результате был сформирован набор исходных данных для логистической регрессии, которая отражала зависимость между вероятностью превышения значения порога и объясняющими переменными $(20,21)$ :

$$
P\left(y\left(x_{i}\right)=1 \mid \varphi_{i}\right)=p_{i}=\frac{1}{1+\exp \left(-\varphi_{i}^{T} \beta\right)},
$$


где $\varphi_{i}-$ факторы, объясняющие фиктивную переменную $y\left(x_{i}\right)$.

В качестве одного из факторов логит-модели использовали набор значений, спрогнозированных методом кригинга. Адекватность построенной логит-модели [2] проверяли с помощью классических приемов статистического анализа - теста Вальда (Wald test, W) и теста отношения правдоподобия (likelihood ratio test, LR) (22).

Для каждой точки исследуемого участка вычисляли вероятность $P(y(x)=1)$, которая при приближении к 1 указывала на превышение значения интересующего параметра порогового уровня $d$, к $0-$ на нахождение значения параметра ниже порога.

Расчеты осуществляли с помощью языка программирования $\mathrm{R}$ (https://www.r-project.org), активно используемого при решении задач точного земледелия (23).

Результаты. На рисунке 1 представлен аэрофотоснимок обследуемого участка поля, а также карта распределения собранных данных (их исходным значениям пропорциональны диаметры кругов), построенная в программе R-statistics. В результате был получен набор значений вегетационного индекса NDVI, величина которого, как известно, коррелирует с содержанием азота в растении в рассматриваемой точке $(24,25)$.

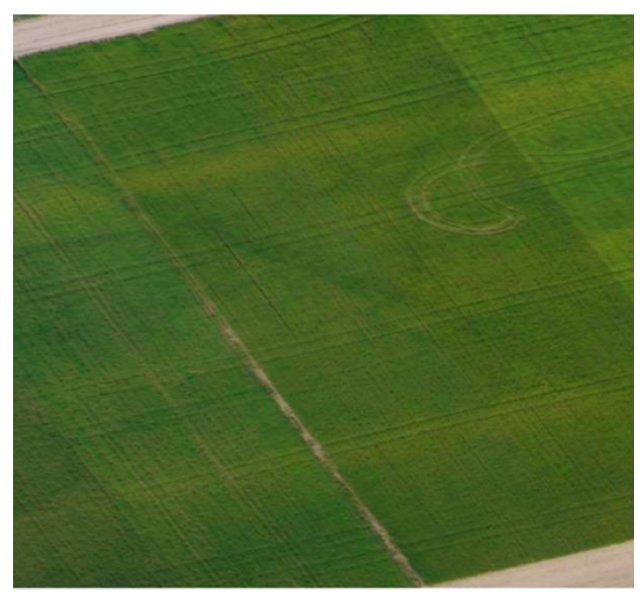

Суть предложенного нами подхода заключается в применении в качестве одного из факторов модели набора спрогнозированных значений, который получали методом кригинга. В соответствии с этим на первом этапе (при прогнозе пространственного распределения интересующего параметра) проверяли выполнение условий геостатистики - стационарности и мультинормальности (26). Обнаруженные выбросы данных отсекали по 2,5\% двусторонним квантилям. Кроме того, оценивали линейную корреля-

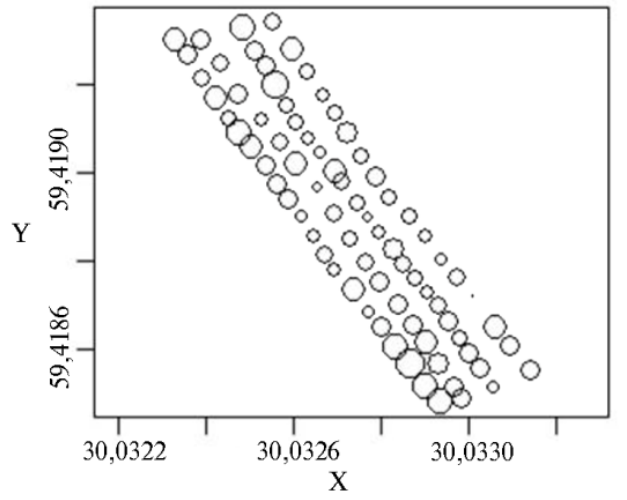

Рис. 1. Аэрофотоснимок исследуемого участка и карта распределения наблюдений (положение на карте): $X$ и $Y-$ координаты наблюдений. Диаметры кругов пропорциональны величине исследуемого показателя NDVI (Normalized Difference Vegetation Index) (д. Меньково, Ленинградская обл., 2015 год). цию интересующего параметра с координатами. В результате пространственный тренд не был выявлен. Проверка с помощью критерия Колмогорова-Смирнова не дала оснований отвергнуть гипотезу о нормальном распределении (достигаемый уровень значимости равен 89,75\%).

На следующем этапе был выполнен вариограммный анализ и построена модель вариограммы с использованием функции vgm. На рисунке 2 представлена полученная экспериментальная вариограмма по четырем направлениям $(0,90$, 135 и $270^{\circ}$ ) с установленной моделью вариограммы. На ее основе проводили ординарный кригинг [1]: из 
ке, после этого осуществляли его прогноз методом кригинга с использованием функции krige.

В результате с помощью функции glm были получены три логитмодели. Величину $d=0,46$ выбрали пороговой. Значимость построенных моделей оценивали критерием отношения правдоподобия. Входные данные для построения логит-моделей были следующими: $N\left(x_{i}\right)$ - значение NDVI в местоположении $x_{i}, i=1,78$; переменная $T=1$, если $N\left(x_{i}\right) \geq 0,46$, иначе $T=0$; переменные $X$ и $Y$ - координаты наблюдений, рассматриваются в качестве объясняющих переменных; $N_{\text {pred }}\left(x_{i}\right)-$ величины параметра, спрогнозированные методом кригинга в наблюдаемых точках.

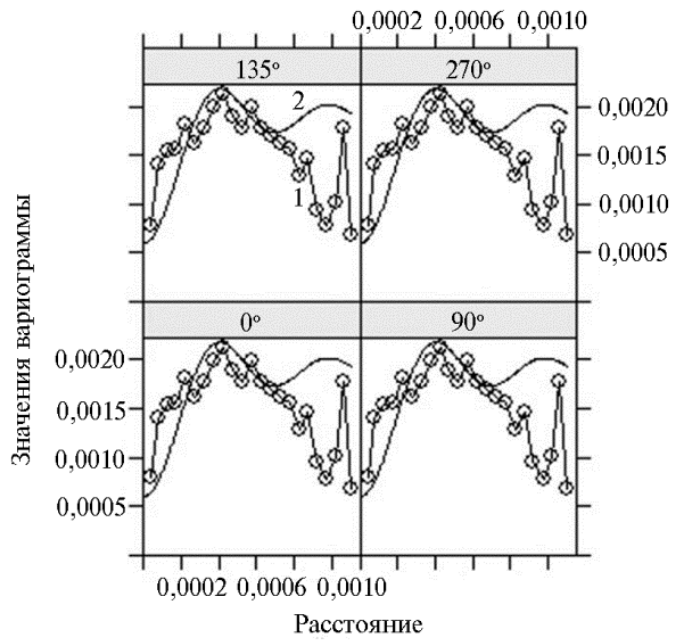

Рис. 2. Экспериментальная вариограмма $\hat{\gamma}(h)$ пространственного распределения показателей NDVI (Normalized Difference Vegetation Index) на исследуемом участке (1) с наложением ее теоретической модели (2) по четырем направлениям (0,90, 135 и 270 $)$ (д. Меньково, Ленинградская обл., 2015 год).

Оценки коэффициентов второй логит-модели была в целом незначимы. Величину $\alpha$ при этом принимали равной 0,05 (табл. 1). Статистический анализ подтвердил, что уравнение этой логит-модели в целом незначимо, в то время как уравнения первой и третьей моделей значимы, при этом их тестирование показало, что третья модель работает лучше.

На следующем этапе для анализа адекватности трех полученных моделей из набора исходных наблюдений поочередно исключали одну точку, строили логит-модели заново и оценивали, как каждая из моделей спрогнозировала вероятность превышения порогового значения в отброшенной точке. Тестирование показало, что первая модель ошибочно определила величину параметра в 26 точках (33,3\%), а третья - в 12 точках (15,38 \%) (табл. 2).

1. Результаты построения логит-моделей пространственного распределения показателей NDVI (Normalized Difference Vegetation Index), построенные с разными объясняющими переменными на основании набора данных аэрофотосъемки (д. Меньково, Ленинградская обл., 2015 год)

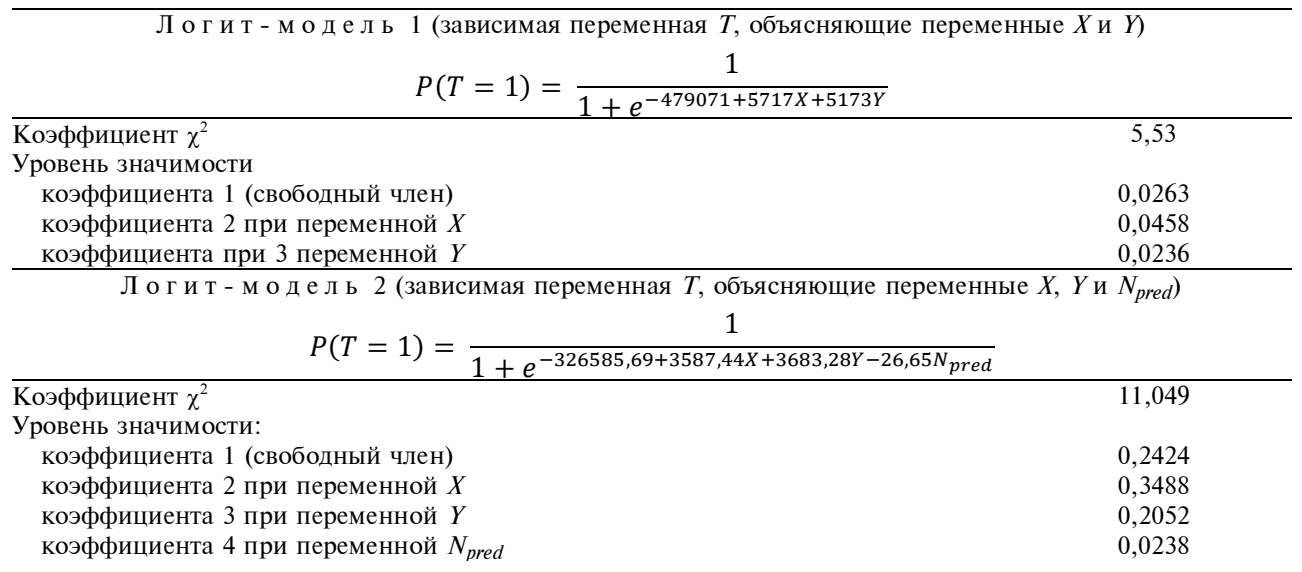




$$
P(T=1)=\frac{1}{1+e^{14,299-32,068 N_{\text {pred }}}}
$$

Уровень значимости:

коэффициента 1 (свободный член) 9,207 коэффициента 2 при переменной $N_{\text {pred }}$ )

П р и м е ч а н и е. В качестве объясняющих переменных при построении моделей методом логистической регрессии взяты координаты наблюдений $X$ и $Y$, а также набор значений $N_{\text {pred }}\left(x_{i}\right)$, спрогнозированных методом кригинга.

2. Выборка из результатов тестирования логит-моделей пространственного распределения показателей NDVI (Normalized Difference Vegetation Index), построенных на основании набора данных аэрофотосъемки (д. Меньково, Ленинградская обл., 2015 год)

\begin{tabular}{l|c|c|c|c|c|c|c}
\hline \multirow{2}{*}{$\begin{array}{l}\text { № точки } \\
\text { кординат }\end{array}$} & $X$ & $Y$ & \multirow{2}{*}{$N$} & $T$ & \multirow{2}{*}{$N_{\text {pred }}$} & \multicolumn{2}{|c}{ Р } \\
\cline { 6 - 8 } & 30,032934 & 59,418484 & 0,527 & 1 & 0,4894404 & $6,187555 \mathrm{e}-11$ & 0,7519646 \\
2 & 30,032902 & 59,418514 & 0,517 & 1 & 0,5037567 & $4,848053 \mathrm{e}-12$ & 0,8326184 \\
3 & 30,032835 & 59,418605 & 0,527 & 1 & 0,4917005 & 0,9999989 & 0,7661754 \\
4 & 30,032695 & 59,418778 & 0,407 & 0 & 0,4396790 & 0,9999876 & 0,3652577 \\
5 & 30,032673 & 59,418811 & 0,455 & 0 & 0,4261240 & $4,863455 \mathrm{e}-12$ & 0,2654931 \\
6 & 30,032588 & 59,418940 & 0,461 & 1 & 0,4387105 & $6,46559 \mathrm{e}-13$ & 0,3366613 \\
7 & 30,032477 & 59,419087 & 0,517 & 1 & 0,4614526 & 0,8979254 & 0,5382949 \\
8 & 30,032327 & 59,419302 & 0,496 & 1 & 0,4600064 & $2,212942 \mathrm{e}-11$ & 0,5256631 \\
9 & 30,032472 & 59,419176 & 0,468 & 1 & 0,4688632 & $2,652915 \mathrm{e}-10$ & 0,6007014 \\
10 & 30.032528 & 59.419119 & 0.411 & 0 & 0,4656420 & $1,943197 \mathrm{e}-06$ & 0,5943595
\end{tabular}

П р и м е ч а и е. $X$ и $Y-$ координаты наблюдений, $N-$ значения исходных наблюдений, $T-$ зависимая переменная, $N_{\text {pred }}\left(x_{i}\right)$ - значения параметра, предсказанные методом кригинга. Для моделей 1 и 3 представлены значения вероятности превышения порога, предсказанные в отброшенных точках наблюдений.

Аналогичные эксперименты, проведенные нами на искусственно смоделированных данных, показали, что вторая полная модель работает лучше в случае, если она статистически значима в целом.

Похожие результаты были получены G.B. Fernandes c соавт. (17), которые изучали логит-модель кредитного скоринга с пространственной переменной в качестве объясняющей. При этом сравнивались две модели: с добавлением пространственной переменной и без нее. Результаты показали, что предложенная авторами методика эффективнее классических. В нашей работе исследовался подход для прогноза пространственного распределения интересующего параметра, основанный на совместном применении кригинга и бинарной регрессии, при этом полная модель (с включением в логит-модель набора величин параметра, спрогнозированных методом кригинга) оказалась лучше альтернативных. Следует отметить, что в России стали применять метод бинарной регрессии в задачах точного земледелия лишь с 2000-х годов. В некоторых сообщениях $(7,20)$ подробно рассматриваются возможности использования логит- и пробит-моделей в растениеводстве, однако пространственная переменная при этом не учитывается.

Таким образом, суть предложенного нами подхода заключается в применении в качестве одного из факторов бинарной регрессии в логитмодели набора значений интересующего параметра, спрогнозированных с использованием ординарного кригинга. В целом сочетание кригинга и бинарной регрессии для оценки состояния растений представляется весьма актуальным и перспективным направлением. Однако при выполнении экспериментов возникали ситуации, когда полная модель была статистически незначима, в связи с чем предложенный подход целесообразно рассмотреть на дополнительных примерах.

1ФГБНУ Агрофизический научно-исследовательский институт,
Поступила в редакцию 22 октября 2018 года 
195220 Россия, г. Санкт-Петербург, Гражданский просп., 14,

e-mail: vlb310154@gmail.com $₫$, apetrushin@agrophys.ru,

mjeka@agrophys.ru, omitrofa@gmail.com;

\title{
2 ФГБОУ ВО Санкт-Петербургский государственный
}

университет,

199034 Россия, г. Санкт-Петербург, Университетская наб., 7/9;

${ }^{3}$ Klaipeda University,

Herkaus Manto 84, LT-92294 Klapeda, Lithuania,

e-mail: vitalij.denisov@ku.lt

Sel'skokhozyaistvennaya biologiya [Agricultural Biology], 2019, V. 54, № 1, pp. 84-90

\section{EXPERIENCE WITH THE USE OF MATHEMATICAL STATISTICS METHODS FOR ASSESSMENT OF AGRICULTURAL PLANTS STATUS}

\author{
V.M. Bure1, 2, A.F. Petrushin', E.P. Mitrofanov' ${ }^{1}$, O.A. Mitrofanova1, 2, V. Denisov ${ }^{3}$
}

${ }^{1}$ Agrophysical Research Institute, 14, Grazhdanskii prosp., St. Petersburg, 195220 Russia, e-mail vlb310154@gmail.com ( $₫$ corresponding author), apetrushin@agrophys.ru, mjeka@agrophys.ru, omitrofa@gmail.com;

${ }^{2}$ Saint Petersburg State University, 7/9, Universitetskaya nab., St. Petersburg, 199034 Russia;

${ }^{3}$ Klaipeda University, Herkaus Manto 84, LT-92294 Klaipeda, Lithuania, e-mail vitalij.denisov@ku.lt ORCID:

Bure V.M. orcid.org/0000-0001-7018-4667

Petrushin A.F. orcid.org/0000-0002-6482-8611

Mitrofanov E.P. orcid.org/0000-0002-1967-5126

Mitrofanova O.A. orcid.org/0000-0002-7059-4727

The authors declare no conflict of interests

Received October 22, 2018

doi: 10.15389/agrobiology.2019.1.84eng

\section{Abstract}

Solving problems related to the assessment of the status of agricultural plants during the growing season, allows us to effectively use fertilizers, obtain favorable yields, improve the quality characteristics of plants, as well as the ecological condition of the field. To solve such problems of precision farming, the use of various methods of mathematical statistics is becoming an increasingly promising direction. The aim of our work was to assess the state of agricultural plants using an approach based on the combined use of kriging and binary regression methods, as well as the determination of nitrogen planting using the NDVI (Normalized Difference Vegetation Index) index. The studies were carried out at the site of an experimental agricultural field located on the territory of the branch of the Agrophysical Institute (Menkovo, Leningrad region) in 2015. With the help of aerial photographs taken from the automatized unmanned aerial vehicle complex Geoscan-401 (Geoscan Group of Companies, Russia), a set of NDVI (Normalized Difference Vegetation Index) vegetation index values was obtained at arbitrary points of the plot. A number of ground-based measurements were also conducted on the studied area of the field. The proposed approach to assessing the state of agricultural plants consisted in the joint use of two methods of mathematical statistics: ordinary kriging and logistic regression. A preliminary variogram analysis was carried out, and a variogram model was constructed. After this, the kriging method was used to calculate a series of predicted values of the parameter being studied. At the next stage, the threshold value of the parameter for the study area was established, and also a dummy variable was entered, taking the value 1 if the parameter value exceeded the threshold, and 0 otherwise. Then a logit model was built, in which one of the factors was a series of estimates of the parameter of interest, obtained using the ordinary kriging method. The input data for building logit models were as follows: $N\left(x_{i}\right)$ is the NDVI value at the location $x_{i}, i=\overline{1.78}$; variable $T=1$, if $N\left(x_{i}\right) \geq 0.46$, otherwise $T=0$; the variables $X$ and $Y$ are the coordinates of the observations, are considered as explanatory variables; $N_{\text {pred }}\left(x_{i}\right)$ is parameter values, predicted using the kriging method at the observed points. All calculations were performed using the $\mathrm{R}$ programming language. As a result of the experiment, three logit models were built with the dependent variable $T$ : in the first model, the explanatory variables $X$ and $Y$; in the second model $-X$, $Y$ and $N_{\text {pred }}$; in the third model $N_{\text {pred }}$. Testing showed that when adding the $N_{\text {pred }}$ variable, the logit model works better ( 2 times less than the erroneous determination of the level of the parameter under study). The results obtained suggest that adding in the binary regression factors a set of values predicted by the kriging method can significantly improve the accuracy of calculations.

Keywords: plant status, Normalized Difference Vegetation Index, NDVI, kriging, binary regression, language $\mathrm{R}$.

\section{REFEREN C ES}

1. Bure V.M., Mitrofanova O.A. Analysis of aerial photographs to predict the spatial distribution of ecological data. Contemporary Engineering Sciences, 2017, 10(4): 157-163 (doi: 10.12988/ces.2017.611175). 
2. Kim Y., Reid J.F., Han S. On-the-go nitrogen sensing and fertilizer control for site-specific crop management. International Journal of Agricultural and Biosystems Engineering, 2006, 7(1): 18-26.

3. Blackmer T.M., Schepers J.S. Aerial photography to detect nitrogen stress in corn. J. Plant Physiol., 1996, 148(3-4): 440-444 (doi: 10.1016/S0176-1617(96)80277-X).

4. Graeff S., Pfenning J., Claupein W., Liebig H.P. Evaluation of image analysis to determine the $\mathrm{N}$-fertilizer demand of broccoli plants. Advances in Optical Technologies, 2008, Article ID 359760 (doi: 10.1155/2008/359760).

5. Thenkabail P.S., Lyon J.G., Huete A. Hyperspectral remote sensing of vegetation. CRC Press, MA, USA, 2011.

6. Franzen D.W., Reitmeier L., Giles J.F., Cattanach A.C. Aerial photography and satellite imagery to detect deep soil nitrogen levels in potato and sugarbeet. Proc. of the $4^{\text {th }}$ International Conference «Precision Agriculture». St. Paul, MN, 1999: 281-290.

7. Bure V.M. Materialy Mezhdunarodnogo seminara, posvyashchennogo pamyati professora Ratmira Aleksandrovicha Poluektova (Poluektovskie chteniya) [Proc. International Seminar dedicated to the memory of Prof. Ratmir Alexandrovich Poluektov (Poluektov reading)]. St. Petersburg, 2014: 118-121 (in Russ.).

8. Debella-Gilo M., Etzelmüller B. Spatial prediction of soil classes using digital terrain analysis and multinomial logistic regression modeling integrated in GIS: Examples from Vestfold County, Norway. Catena, 2009, 77(1): 8-18 (doi: 10.1016/j.catena.2008.12.001).

9. Kempen B., Brus D.J., Heuvelink G.B.M., Stoorvogel J.J. Updating the 1:50,000 Dutch soil map using legacy soil data: a multinomial logistic regression approach. Geoderma, 2009, 151(34): 311-326 (doi: 10.1016/j.geoderma.2009.04.023).

10. Yakushev V.P., Zhukovskii E.E., Kabanets A.L., Petrushin A.F., Yakushev V.V. Variogrammnyi analiz prostranstvennoi neodnorodnosti sel'skokhozyaistvennykh polei dlya tselei tochnogo zemledeliya [Variogram analysis of agricultural fields spatial heterogeneity for precision farming]. St. Petersburg, 2010 (in Russ.).

11. Claret M.M., Urrutia R.P., Ortega R.B., Best S.S., Valderrama N.V. Quantifying nitrate leaching in irrigated wheat with different nitrogen fertilization strategies in an Alfisol. Chilean Journal of Agricultural Research, 2011, 71(1): 148-156 (doi: 10.4067/S0718-58392011000100018).

12. Krasilnikov P., Sidorova V. Geostatistical analysis of the spatial structure of acidity and organic carbon in zonal soils of the Russian plain. In: Soil geography and geostatistics: concepts and applications. P. Krasilnikov, F. Carré, L. Montanarella (eds.). European Communities, Luxembourg, 2008: 55-67.

13. Dulaney W.P., Lengnick L.L., Hart G.F. Use of geostatistical techniques in the design of an agricultural field experimental. Proc. of the Survey research methods section. Alexandria, VA, 1994: 183-187.

14. Adamsen F.J., Pinter P.J., Barnes E.M. Measuring wheat senescence with a digital camera. Crop Science, 1999, 39(3): 719-724 (doi: 10.2135/cropsci1999.0011183X003900030019x).

15. Panayi E., Peters G.W., Kyriakides G. Statistical modelling for precision agriculture: a case study in optimal environmental schedules for Agaricus Bisporus production via variable domain functional regression. PLoS ONE, 2017, 12(9): e0181921 (doi: 10.1371/journal.pone.0181921).

16. Isaaks E., Srivastava M. An introduction to applied geostatistics. Oxford University Press, NY, USA, 1989.

17. Fernandes G.B., Artes R. Spatial dependence in credit risk and its improvement in credit scoring. Eur. J. Oper. Res., 2016, 249(2): 517-524 (doi: 10.1016/j.ejor.2015.07.013).

18. Dem'yanov V., Savel'eva E. Geostatistika. Teoriya i praktika [Geostatistics. Theory and practice]. Moscow, 2010 (in Russ.).

19. Webster R., Oliver M.A. Geostatistics for environmental scientists, Second edition. John Wiley and Sons Ltd, Chichester, UK, 2007 (doi: 10.1002/9780470517277).

20. Yakushev V.P., Bure V.M., Parilina E.M. Binarnaya regressiya i ee primenenie $v$ agrofizike [Binary regression and its application in agrophysics]. St. Petersburg, 2015 (in Russ.).

21. Hosmer D., Lemeshow S. Applied logistic regression, Second edition. Wiley, NY, 2000 (doi: 10.1002/0471722146).

22. Bure V.M., Parilina E.M. Teoriya veroyatnostei i matematicheskaya statistika: uchebnoe posobie [Probability theory and mathematical statistics: tutorial]. St. Petersburg, 2013 (in Russ.).

23. Plant R.E. Spatial data analysis in ecology and agriculture using R. CRC Press, Boca Raton, 2012.

24. Yakushev V.P., Kanash E.V., Konev A.A., Kovtyukh S.N., Lekomtsev P.V., Matveenko D.A., Petrushin A.F., Yakushev V.V., Bure V.M., Rusakov D.V., Osipov Yu.A. Teoreticheskie $i$ metodicheskie osnovy vydeleniya odnorodnykh tekhnologicheskikh zon dlya differentsirovannogo primeneniya sredstv khimizatsii po opticheskim kharakteristikam poseva: prakticheskoe posobie [Theoretical and methodological basis for the allocation of homogeneous technological zones for the differentiated use of chemicals according to the optical characteristics of sowing: practical guide]. St. Petersburg, 2010 (in Russ.).

25. Kanash E.V., Osipov Ju.A. Optical signals of oxidative stress in crops physiological state diagnostics. Proc. $7^{\text {th }}$ European conference on precision agriculture. Wageningen, Netherlands, 2009: 81-89.

26. Goovaerts P. Geostatistics for natural resources evaluation. Oxford University Press, NY, 1997. 